\title{
The role of imaging techniques in the diagnosis, staging and choice of therapeutic conduct in pregnancy associated breast cancer
}

\author{
Iulian Gabriel Goidescu', Georgiana Nemeti ${ }^{1}$, Gabriela Caracostea ${ }^{1}$, Dan Tudor Eniu ${ }^{2}$, \\ Angelica Chiorean ${ }^{3}$, Roxana Pintican ${ }^{4}$, Gheorghe Cruciat ${ }^{1}$, Daniel Mureșan ${ }^{1}$
}

${ }^{1}$ Department of Obstetrics and Gynecology, "Iuliu Hațieganu" University of Medicine and Pharmacy, ${ }^{2}$ Department of Surgical Oncology and Oncological Gynecology, "Iuliu Haţieganu” University of Medicine and Pharmacy, ${ }^{3}$ Medimages Breast Center, Cluj-Napoca, Department of Radiology and Medical Imaging, County Hospital Cluj-Napoca, Romania

\begin{abstract}
Breast cancer diagnosed during pregnancy is at increasing incidence due to the increased frequency of obesity, the postponement of the first pregnancy to later decades of life and the advances of diagnostic techniques. Clinical and imaging diagnosis is difficult during gestation due to adaptive changes of the maternal organism, the mammary glads in particular. Furthermore, the therapeutic approach is limited both by the possible side effects on the fetus and by the skepticism of the couple over these therapeutic regimens.

The present paper aims to review the main diagnostic steps to confirm pregnancy associated breast cancer, as well as the therapeutic possibilities during this period, related to the potential adverse effects concerning pregnancy.

Keywords: pregnancy associated breast cancer (PABC); chemotherapy; pregnancy; breast ultrasound; magnetic resonance imaging
\end{abstract}

\section{Introduction}

Breast cancer is the most commonly diagnosed neoplasia in women worldwide, with an incidence of 94.2/100,000 in Europe [1]. There is a notable increase in the frequency of breast cancer with age. Therefore, given the current trend of postponing the first pregnancy towards the 4th decade of life, the diagnosis of breast cancer during pregnancy is expected to rise in the future [2]. Its estimated incidence is of approximately 1 in 3000

Received 21.03.2019 Accepted 23.05.2019

Med Ultrason

2019, Vol. 21, No 3, 336-343

Corresponding author: Georgiana Nemeti

Department of Obstetrics and Gynecology

3-5 Clinicilor stret, 400006 Cluj-Napoca

E-mail: georgiana_nemeti@yahoo.com

Phone: +40 $7235 \overline{6} 5256$ pregnant women, being the second most common malignancy in pregnancy [3] and accounting for approximately $1 \%$ of all breast cancers [4].

Pregnancy-associated breast cancer (PABC) is defined as breast cancer diagnosed during pregnancy or during the first year after delivery [3]. Physiological changes occurring within the breast during pregnancy may delay diagnosis [5].

The majority of PABCs usually occur in young women, under 40 years of age, with a high-risk genetic profile [6]. Most patients are carriers of pathogenic mutations $[6,7]$, especially breast cancer susceptibility genes (BRCA), which makes genetic testing mandatory in all these patients [8]. From a different standpoint, carriers of pathogenic mutations who bear children have a significantly increased risk to develop breast cancer by the age of 40 as opposed to carriers who are nulliparous, with every new pregnancy increasing this risk [9]. 
Establishing the diagnosis of cancer in pregnancy is a challenge for physicians, but communicating with patients and their families is even more difficult. Patients, their families and their physicians have a burdening decision to make, whether to terminate the pregnancy and follow the required treatment or, on the other hand, to postpone therapy until after delivery [10].

PABCs are highly aggressive being often undifferentiated, hormone-independent (estrogen receptor negative ER-, progesterone receptor negative PR-) and HER (human epidermal growth factor receptor) positive tumors, in up to one third of the cases $[6,11]$.

Clinical management of these patients is challenging and must be adjusted according to the gestational age, fetal viability and the couple's wishes [12]. The benefits and risks of the different diagnostic and therapeutic options must be carefully weighed and optimal treatment should be offered to the mother with minimal risk of harm to the fetus.

In this paper we aim to review the main diagnostic steps to confirm PABC, as well as the therapeutic possibilities during this period, related to the potential adverse effects on the pregnancy.

\section{Diagnosis}

$\mathrm{PABC}$ is a difficult diagnosis to make due to the possible misleading aspect of physiologic breast changes during pregnancy caused by the new hormonal status and because certain investigations are not allowed during some periods of pregnancy.

\section{Clinical examination}

Clinical examination of both mammary regions and axillae should be mandatory at the first prenatal visit and any palpable nodule in this region, bloody nipple discharge or focal pain should be further investigated using breast ultrasound. Small tumors are difficult to be detected and nodules in the breast are often overlooked or presumed to be benign proliferative changes. This leads to a delay in diagnosis and the finding of more advanced stages [5].

\section{Diagnostic imaging}

Detection rates of PABC are very high using mammography, ultrasonography (US) or magnetic resonance (MRI) being 91\%, 100\% and 97\%, respectively, according to Myers et al [13].

Breast US represents the most appropriate and safe imaging tehnique for evaluating breast disorders in women during pregnancy and lactation [14]. Grey scale US has a high sensitivity and specificity for the diagnosis of PABC, can differentiate cystic from solid breast lesions and is considered to be the golden standard for palpable breast masses during pregnancy [15] (fig 1).
In a study conducted on 22 patients diagnosed with breast cancer during pregnancy or lactation, Ahn et al [16] showed that US had a sensitivity of $100 \%$, data supported by other studies $[15,17]$. Based on this evidence, the National Comprehensive Cancer Network (NCCN) recommends the use of US for the detection of both breast masses and loco-regional adenopathies [9].

Color Doppler US and elastography are complementary to the basic US examination and provide important details which serve to refine the diagnosis [18,19] (fig 2, $3)$.

Mammography is a useful imaging tool in the workup of PABC but it can be used after the first trimester of pregnancy and only with abdominal shield protection of the patient [2]. The level of radiation achieved by a bilateral mammogram is of approximately $3 \mathrm{mGy}$, the uterus being exposed to less than $0.03 \mu \mathrm{Gy}$. This exposure dose is lower than the known teratogenic load, which is 50 $\mathrm{mGy}$ [15]. The accuracy of mammography in pregnancy can reach up to $80 \%$ [20] due to the high vascularization of the breast, which may increase the false negative results rate [21]. However, sometimes mammography can bring valuable additional information, because density changes of breast parenchyma during pregnancy and lactation occur to different degrees [21].

Breast MRI has a sensitivity of $98 \%$ [13]. Contrastenhanced MRI is not recommended during the first trimester of pregnancy because the contrast agent used, gadolinium, is classified as pregnancy category C. Gadolinium-based contrast media cross the placenta in measurable quantities and can be identified in the amniotic fluid [22].

Gadolinium MRI can be associated with an increased risk of rheumatological, inflammatory or infiltrative skin disorders during childhood and stillbirth/neonatal death during late pregnancy and postpartum $[15,23]$. For this reason, breast MRI or staging MRI using gadoliniumbased contrast agents should be avoided during pregnancy $[15,23]$. The use of contrast-enhanced exposure can be safely performed during lactation [15].

MRI examination is superior to other imaging tools in identifying multicentric disease which cannot be detected by mammography or US [13] but must be used with caution during pregnancy (fig 4).

\section{Breast biopsy}

Breast biopsy is the most accurate investigation that confirms and establishes the final diagnosis, both histologically and immunohistochemically. It is preferable to use core needle biopsy rather than incisional biopsy [3] and to avoid fine needle aspiration (FNA) due to the increased false-positive false-negative rates [3]. 


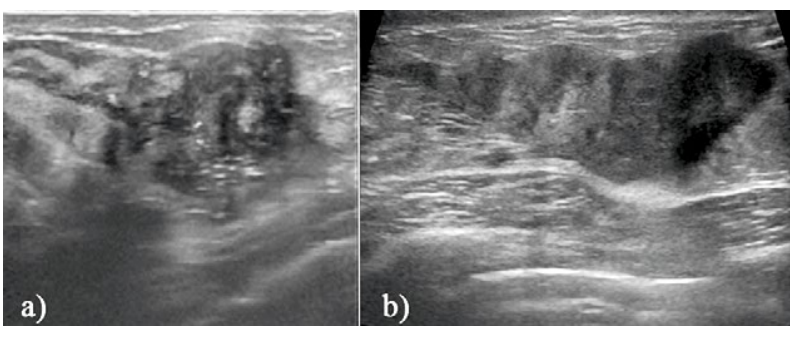

Fig 1. 2D ultrasound images of malignant breast tumors: a) 25 year old patient, 13 weeks pregnancy, palpable mass in the right breast; b) 38 year old patient, 8 weeks pregnancy, palpable mass in the right breast.

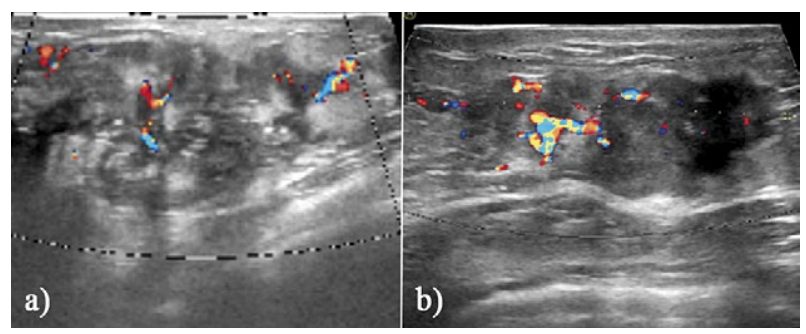

Fig 2. Color Doppler breast ultrasound in pregnancy: a) 25 year old patient, 13 weeks pregnancy, palpable mass in the right breast; b) 38 year old patient, 8 weeks pregnancy, palpable mass in the right breast.
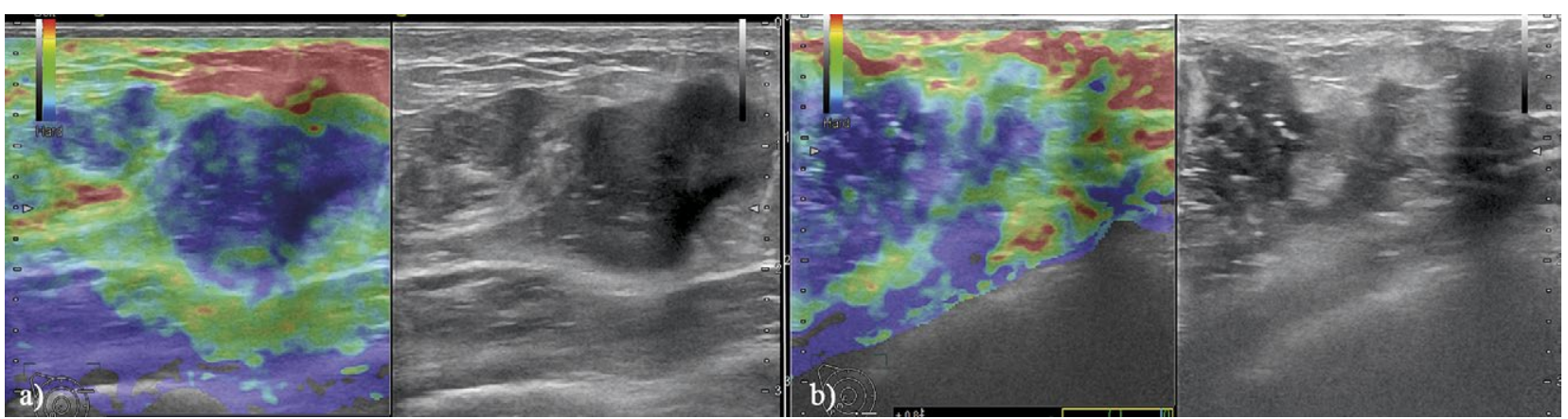

Fig 3. Breast sonoelastography in pregnancy: a) 25 year old patient, 13 weeks pregnancy, palpable mass in the right breast; b) 38 year old patient, 8 weeks pregnancy, palpable mass in the right breast.

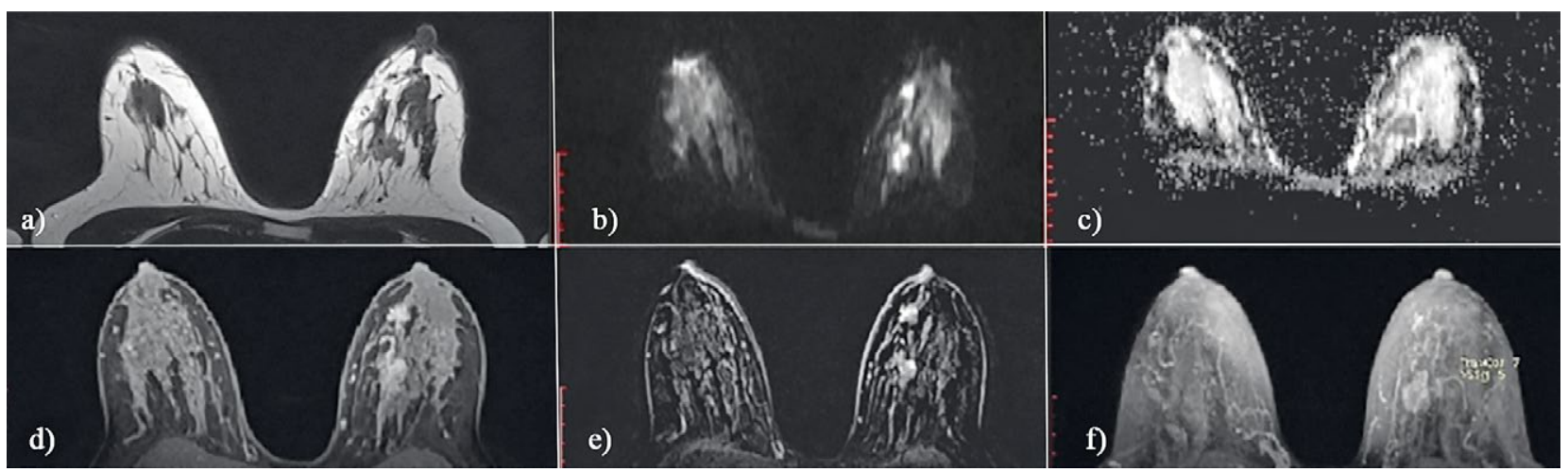

Fig 4. Breast MRI: a) in the upper-inner quadrant of the left breast there are two T2 isointense masses with irregular margins and restricted diffusion on DWI / ADC (bc) sequences; intense early contrast enhancement and washout on delay phases - Kuhl type III curve - is seen on T1 FatSat postcontrast enhancement, Substraction T1 FatSat and MIP sequences (d,e,f).

US guided core biopsy is considered the golden standard procedure for assessing breast tumors during pregnancy and lactation. It is quite a safe procedure, the most commonly encountered complications being the risk of bleeding, milk fistula and infection. These risks can be decreased by breastfeeding cessation before biopsy or the use of compressive hemostasis [24].

Unfortunately, PBACs generally have larger dimensions and are more aggressive forms, similar to those seen in younger women or in mutation carriers [25]. The most common histological and immunohistochemical features associated with pregnancy are high-grade tumors, triple negative molecular forms, with a high rate of lymph-vascular space involvement $[11,26]$.

\section{Staging}

Ultrasound (+/-Doppler) examination may be used without restriction throughout pregnancy, being the investigation of choice for pelvic and abdominal staging [10]. 
Chest X-ray - may be considered safe as long as a protective shield is used, especially in the second and third trimester of pregnancy. The fetus is exposed to a dose ranging from 0 to 0.0001 Gy [15].

$M R I$ - Exposure to MRI during pregnancy was not associated with increased risk of harm to the fetus or in early childhood. However contrast agents should be used with caution and only after the first trimester of pregnancy due to the risk of complications [15,23].

Computer tomography (CT) should not be withheld if the benefit can outweigh the risks, but should be avoided when we can use other methods such as MRI [15].

Positron emission tomography-CT must be avoided if the couple decides to keep the pregnancy [10]. There are some studies that advocate the use of fluorodeoxyglucose-F18 PET-CT $\left({ }^{18} \mathrm{~F}-\mathrm{FDG}\right.$ PET-CT) in pregnant women with cancer, when medically necessary, arguing that the fetal exposure is low. Larger randomized trials are required to certify the safety of these methods in pregnancy $[27,28]$.

\section{Treatment options}

Treatment options for patients with PABC are similar to those suitable for nonpregnant patients, with the same basic principles: local disease control and prevention of systemic extension, figure 5 [29]. During pregnancy certain therapeutic agents should be avoided and in the decision to adopt a certain drug regimen the following should be taken into account: the couple's desire for keeping the pregnancy, gestational age at treatment onset, the absence of fetal abnormalities at routine genetic or ultrasound screening tests, fetal viability [29] (fig 5).

\section{Surgery}

Primary surgery addresses particularly early stages of $\mathrm{BC}$, with small tumors and absent loco regional adenopathies, or for situations when the patient refuses neoadjuvant treatment [29]. Selection of these patients who benefit most from primary surgery is done using imaging methods such as US and MRI, which evaluate disease extension [15,29].

Surgery for breast cancer is safe and it can be performed in all trimesters of pregnancy with minimal risks for the fetus. According to the NCCN guidelines there are two possible surgical approaches in breast cancer: either radical modified mastectomy (RMM) or conservative surgery in early stages - lumpectomy with axillar lymph node dissection (ALN) or sentinel lymph node biopsy (SLNB) [24]. The rationale behind the choice of the surgical procedure is the need for adjuvant radiotherapy after conservative interventions, which is to be avoided during pregnancy. This is why surgeons resort to minimally invasive procedures performed only in the $3^{\text {rd }}$ trimester of pregnancy [30,31].

RMM with axillary lymph node dissection is considered to be the best choice for PABC patients diagnosed during the first two trimesters of pregnancy who opt for continuation of pregnancy [30]. Several studies concluded that surgical treatment is usually well accepted by patients compared to chemotherapy due to the relatively low risk of complications for both the mother and fetus [25,31].

Survival rates are similar for both surgical techniques. SLNB is safer than ALN in pregnant women with a better outcome of recovery due to less intra/postoperative bleeding and reduced duration of surgery [30].

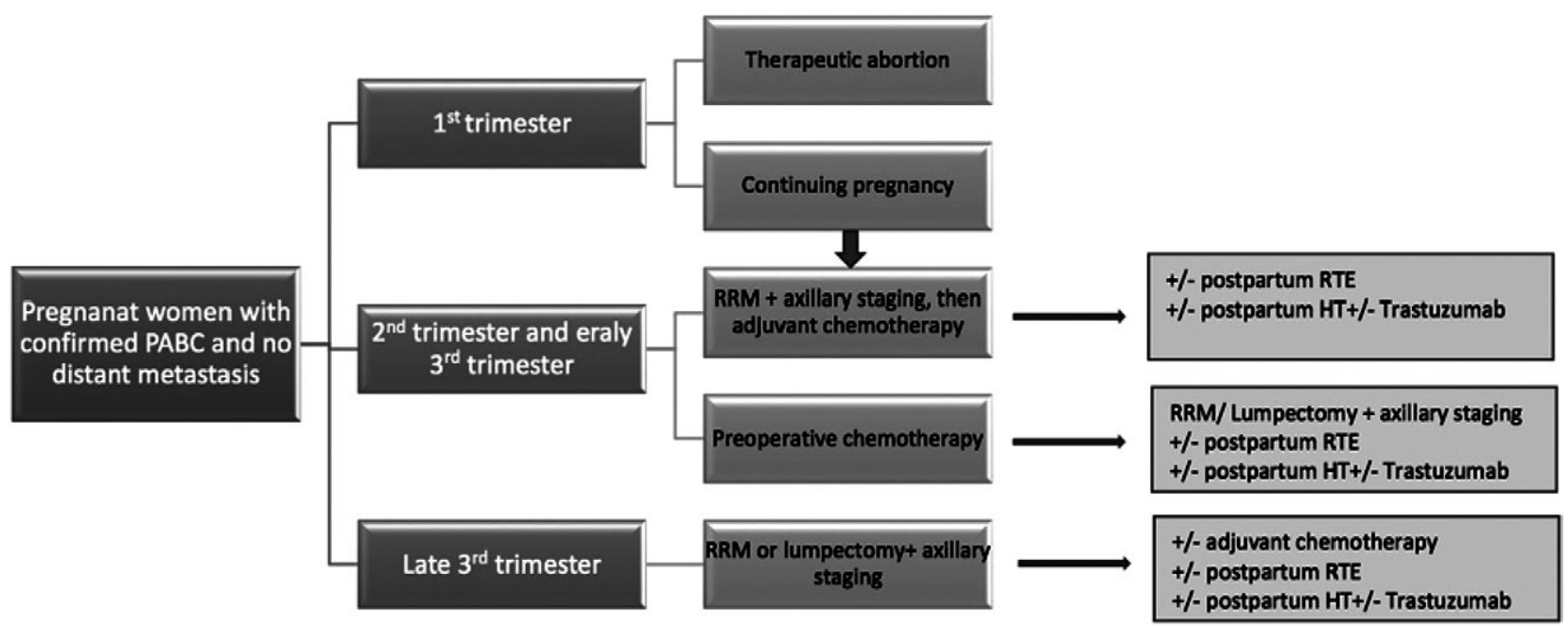

Fig 5. NCCN 2018 Guidelines - Breast cancer during pregnancy ([1], adapted). 
Lymphoscintigraphy with Tc99 (technetium 99) for SNLB during pregnancy performed to avoid ALN is considered to be safe, with low levels of remote radiation. The dose absorbed by the fetus is of approximately $4.3 \mathrm{mGy}$, which is below the $50 \mathrm{mGy}$ threshold thought to induce fetal adverse effects, and can be performed safely during the $3^{\text {rd }}$ trimester [15,31].

\section{Radiotherapy}

Radiation therapy is contraindicated during pregnancy due to its teratogenic consequences $[15,25]$. Several adverse effects were reported such as intrauterine growth restriction, stillbirth, mental retardation, childhood cancers. These effects depend on the duration of radiation and the total dose of exposure $[15,25]$.

\section{Chemotherapy}

When breast tumors are large, ultrasound indicates axillary or supraclavicular lymph node involvement, or gestational age does not allow surgery as a primary treatment. Neoadjuvant chemotherapy is preferred, because hormonal therapy and target therapy with monoclonal antibodies are not allowed during this period [15].

Chemotherapy is employed as adjuvant or neoadjuvant treatment. Because of its proven teratogenicity, it is best avoided during the first trimester of pregnancy [10]. When used during the period of organogenesis it has been cited to cause fetal malformations and spontaneous abortion (ranging from 10-20\%) [10]. Later in pregnancy chemotherapy does not appear to significantly increase the risk of malformations.

After 14 weeks of pregnancy the possibility of such complications decreases to an acceptable level (between $1.5-3 \%)$ [32].

According to the NCCN 2018 Diagnostic and Treatment Guide for Breast Cancer, the risk of malformations in children from chemotherapy-borne pregnancies is even smaller, around $1.3 \%$, similar to that of the general population [24].

These risks after in-utero exposure to chemotherapy during late pregnancy are reduced to: low birthweight, subtle changes to heart function due to cardiomyopathy caused by anthracyclines [33,34], respiratory distress syndrome [35], fetal anemia [35], bilateral reduction of hearing and mild ventriculomegaly $[35,36]$. Also, during childhood there may be dental problems, because tooth formation is completed only postnatally, and infants can be more susceptible to dental malformations and dental caries [36]. Neurocognitive problems during childhood such as disturbances in intelligence, memory and attention deficits and language impairment [36] were also described but there is a lack of evidence to support such an association.

Chemotherapy dosage during pregnancy is complicated due to maternal pregnancy induced adaptive mechanisms such as increased plasma volume, increased hepatic metabolism, increased renal excretory function, decreased albumin levels and decreased gastro-intestinal motility [10].

Chemotherapeutic agents most commonly used in pregnancy are anthracyclines and alkylating agents. Furthermore, the use of the FAC regimen (5FU, Doxorubicin, Cyclophosphamide) appears to be associated with a low risk of complications and fetal malformations when administered in the $2^{\text {nd }}$ and $3^{\text {rd }}$ trimesters of pregnancy $[4,37]$.

Anthracyclines are topoisomerase inhibitors which may be used as first-line chemotherapy in the treatment of breast cancer in combination with Cyclophosphamide [10]. According to Cardonick et al [38], it is preferable to use Doxorubicin rather than Epirubicin, the latter having more adverse effects. Fetal cardiotoxicity due to in utero exposure to anthracyclines reported by other studies $[4,39]$, was ruled out by subsequent research which assessed in utero cardiac function and postpartum newborn cardiac function $[3,40]$.

Taxanes may also be administered in pregnancy, but data regarding their safety is reduced. If used, the weekly administration of Paclitaxel is preferred [5,41]. Recent studies advocate the effectiveness and safety of taxanes administration during pregnancy with relatively low side effects rates $[42,43]$. Possible consequences are mild intrauterine growth restriction, prematurity, mild hydrocephaly, neonatal respiratory distress syndrome and gastroesophageal reflux [43]. The same study reports the delivery of completely healthy neonates in $76.7 \%$ of cases and healthy children in $90 \%$ of cases at 16 months follow-up [43].

\section{Hormonal therapy}

Neither tamoxifen, nor aromatase inhibitors administration is recommended during pregnancy due to their teratogenic effects $[2,25]$. Some studies reported up to $20 \%$ risk of fetal abnormalities in patients using hormonal therapy during pregnancy, such as craniofacial malformations or ambiguous genitalia [44].

\section{Trastuzumab therapy}

Human monoclonal antibodies are recommended for the treatment of patients with HER2-positive immunohistochemistry, localized breast cancer, following surgery [45]. Trastuzumab targets the human epidermal growth factor receptor which is expressed in many embryonal tissues in normal pregnancies [46]. Literature reports fetal kidney injury after monoclonal antibody administration with subsequent occurrence of oligohydramnios/anhydramnios, and for these reasons it can only be used as postpartum adjuvant treatment [46]. 


\section{Termination of pregnancy}

Termination of pregnancy in the $1^{\text {st }}$ trimester can be considered for advanced stages of breast cancer, if the woman has children or if the couple considers this option $[40,47]$.

In the $2^{\text {nd }}$ and $3^{\text {rd }}$ trimester termination of pregnancy and delayed postpartum treatment are not valid options, because neither leads to improved survival rates or prognosis [47].

\section{Follow up and neoadjuvant treatment monitoring}

Follow up of the tumor response to neoadjuvant chemotherapy is mainly achieved by US, MRI being recommended especially multicentric breast disease [24,25].

Monitoring treatment response and disease progression is primarily achieved using US and/or MRI. This follow up can be improved by using tumor markers such as CA15-3 [10] or blood serum free amino acid profiles, but the main disadvantage of the latter is that changes in the protein metabolism during pregnancy can cause misinterpretation of the results [48].

\section{Maternal prognosis}

Although there were studies which recorded similar prognosis for PABC and breast cancer in non pregnant women matched for age and stage [49], an important body of research showed poorer survival rates in patients with PABC [47,50-52]. Callihan et al showed that breast cancer diagnosed within five-years postpartum has a 2.8 times higher risk for metastasis and a 2.7 times higher mortality risk compared to breast cancer diagnosed in nulliparous patients [52].

Usually, breast cancers found in women of a younger age are more aggressive than those diagnosed in older women and are frequently associated with the presence of pathogenic mutations [6]. Therefore, breast cancer in pregnancy is generally more aggressive and is detected in more advanced stages compared to non-pregnant women [6].

There have been literature reports of placental metastases in rare situations. The assessment of placental histology after delivery in all cases of PABC is mandatory $[53,54]$.

\section{Conclusions}

Breast cancer in pregnancy is an uncommon condition with detrimental consequences for the patient, the family and physicians. The therapeutic options and their sequence must be pondered by a complex interdiscipli- nary team consisting of an oncologist, a maternal-fetal medicine specialist and an oncologic surgeon, after counseling of the couple and in agreement with their wishes.

US is the safest imaging option in pregnant women but mammography and breast MRI can be performed if necessary. Core needle biopsy is mandatory to confirm the presence of disease and to establish the molecular subtype, which then dictates the management sequence.

The therapeutic conduct and staging are decided mainly using imaging examinations, such as US or MRI, but if CT is necessary, it should not be excluded from the investigation panel.

RMM with axillary lymph node dissection is the surgical treatment of choice for women with early stage breast cancer, but conservative surgery can be a suitable option for selected cases, especially in the third trimester of pregnancy.

Chemotherapy may be used in pregnancy after the first trimester but hormonal therapy, monoclonal antibodies and radiotherapy are best avoided during pregnancy due to their high risk of fetal complications.

\section{Conflict of interest: none}

\section{References}

1. Senkus E, Kyriakides S, Ohno S, et al. Primary breast cancer: ESMO Clinical Practice Guidelines for diagnosis, treatment and follow-up. Ann Oncol 2015;26 Suppl 5:v8v30.

2. Loibl S, Von Minckwitz G, Gwyn K, et al. Breast carcinoma during pregnancy: International recommendations from an expert meeting. Cancer 2006;106:237-246.

3. Salani R, Billingsley CC, Crafton SM. Cancer and pregnancy: An overview for obstetricians and gynecologists. Am J Obstet Gynecol 2014;211:7-14.

4. Loibl S, Han SN, von Minckwitz G, et al. Treatment of breast cancer during pregnancy: An observational study. Lancet Oncol 2012;13:887-896.

5. Garcia-Manero M, Royo MP, Espinos J, Pina L, Alcazar JL, Lopez G. Pregnancy associated breast cancer. Eur J Surg Oncol 2009;35:215-218.

6. Goidescu IG, Eniu DT, Caracostea GV, Cruciat G, Stamatian F. Associations of pathogenic mutations responsible for breast cancer risk with histology and immunohistochemistry in Romanian population. Rev Romana Med Lab 2018;26:165-175.

7. Goidescu IG, Caracostea G, Eniu DT, Stamatian FV. Prevalence of deleterious mutations among patients with breast cancer referred for multigene panel testing in a Romanian population. Clujul Med 2018;91:157-165.

8. Keyser EA, Staat BC, Fausett MB, Shields AD. Pregnancyassociated breast cancer. Rev Obstet Gynecol 2012;5:94-99.

9. NCCN. NCCN Clinical Practice Guidelines in Oncology (NCCN Guidelines $\left.{ }^{\circledR}\right)$ Breast Cancer. Version 2.2017. 2017;1-191. 
10. Amant F, Halaska MJ, Fumagalli M, et al. Gynecologic cancers in pregnancy: guidelines of a second international consensus meeting. Int J Gynecol Cancer 2014;24:394-403.

11. Middleton LP, Amin M, Gwyn K, Theriault R, Sahin A. Breast carcinoma in pregnant women: assessment of clinicopathologic and immunohistochemical features. Cancer 2003;98:1055-1060.

12. Loibl S, Schmidt A, Gentilini O, et al. Breast Cancer Diagnosed During Pregnancy: Adapting Recent Advances in Breast Cancer Care for Pregnant Patients. JAMA Oncol 2015; 1:1145-1153.

13. Myers KS, Green LA, Lebron L, Morris EA. Imaging Appearance and Clinical Impact of Preoperative Breast MRI in Pregnancy-Associated Breast Cancer. AJR Am J Roentgenol 2017;209:W177-W183.

14. Ayyappan AP, Kulkarni S, Crystal P. Pregnancy-associated breast cancer: spectrum of imaging appearances. Br J Radiol 2010;83:529-534.

15. Committee on Obstetric Practice. Committee Opinion No. 723: Guidelines for Diagnostic Imaging During Pregnancy and Lactation. Obstet Gynecol 2017;130:e210-e216.

16. Ahn BY, Kim HH, Moon WK, et al. Pregnancy- and lactation-associated breast cancer: Mammographic and sonographic findings. J Ultrasound Med 2003;22:491-497.

17. Robbins J, Jeffries D, Roubidoux M, Helvie M. Accuracy of diagnostic mammography and breast ultrasound during pregnancy and lactation. AJR Am J Roentgenol 2011;196:716-722.

18. Kim MY, Cho N, Yi A, Koo HR, Yun BL, Moon WK. Sonoelastography in distinguishing benign from malignant complex breast mass and making the decision to biopsy. Korean J Radiol 2013;14:559-567.

19. Chiorean AR, Szep MB, Feier DS, Duma M, Chiorean MA, Strilciuc S. Impact of Strain Elastography on BI-RADS classification in small invasive lobular carcinoma. Med U1trason 2018;20:148-153.

20. Yang WT, Dryden MJ, Gwyn K, Whitman GJ, Theriault R. Imaging of breast cancer diagnosed and treated with chemotherapy during pregnancy. Radiology 2006;239:52-60.

21. Rimawi BH, Green V, Lindsay M. Fetal implications of diagnostic radiation exposure during pregnancy: Evidencebased recommendations. Clin Obstet Gynecol 2016;59:412418.

22. Vashi R, Hooley R, Butler R, Geisel J, Philpotts L. Breast imaging of the pregnant and lactating patient: imaging modalities and pregnancy-associated breast cancer. AJR Am J Roentgenol 2013;200:321-328.

23. Ray JG, Vermeulen MJ, Bharatha A, Montanera WJ, Park AL. Association Between MRI Exposure During Pregnancy and Fetal and Childhood Outcomes. JAMA 2016;316:952961.

24. Gradishar WJ, Anderson BO, Balassanian R, et al. Breast Cancer, Version 4.2017, NCCN Clinical Practice Guidelines in Oncology. J Natl Compr Canc Netw 2018;16:310320.

25. Peccatori FA, Azim HA Jr, Orecchia R, et al. Cancer, pregnancy and fertility: ESMO Clinical Practice Guidelines for diagnosis, treatment and follow-up. Ann Oncol 2013;24 Suppl 6:vi160-vi170.

26. Barnes DM, Newman LA. Pregnancy-associated breast cancer: a literature review. Surg Clin North Am 2007;87:417430 .

27. Zanotti-Fregonara P, Koroscil TM, Mantil J, Satter M. Radiation dose to the fetus from [(18)F]-FDG administration during the second trimester of pregnancy. Health Phys 2012;102:217-219.

28. Takalkar AM, Khandelwal A, Lokitz S, Lilien DL, Stabin MG. 18F-FDG PET in pregnancy and fetal radiation dose estimates. J Nucl Med 2011;52:1035-1040.

29. Rovera F, Chiappa C, Coglitore A, et al. Management of breast cancer during pregnancy. Int J Surg 2013;11 Suppl 1:S64-S68

30. Gentilini O, Cremonesi M, Toesca A, et al. Sentinel lymph node biopsy in pregnant patients with breast cancer. Eur J Nucl Med Mol Imaging 2010;37:78-83.

31. Han SN, Amant F, Cardonick EH, et al. Axillary staging for breast cancer during pregnancy: feasibility and safety of sentinel lymph node biopsy. Breast Cancer Res Treat. 2018;168:551-557.

32. McGrath SE, Ring A. Chemotherapy for breast cancer in pregnancy: evidence and guidance for oncologists. Ther Adv Med Oncol 2011;3:73-83.

33. Amant F, Van Calsteren K, Halaska MJ, et al. Longterm cognitive and cardiac outcomes after prenatal exposure to chemotherapy in children aged 18 months or older: an observational study. Lancet Oncol 2012;13:256264.

34. Cardonick EH, Gringlas MB, Hunter K, Greenspan J. Development of children born to mothers with cancer during pregnancy: comparing in utero chemotherapy-exposed children with nonexposed controls. Am J Obstet Gynecol 2015;212:658.e1-e8.

35. Hahn KM, Johnson PH, Gordon N, et al. Treatment of pregnant breast cancer patients and outcomes of children exposed to chemotherapy in utero. Cancer 2006;107:12191226.

36. Vandenbroucke T, Verheecke M, Fumagalli M, Lok C, Amant F. Effects of cancer treatment during pregnancy on fetal and child development. Lancet Child Adolesc Health 2017;1:302-310.

37. Framarino-Dei-Malatesta M, Sammartino P, Napoli A. Does anthracycline-based chemotherapy in pregnant women with cancer offer safe cardiac and neurodevelopmental outcomes for the developing fetus? BMC Cancer 2017;17:777.

38. Cardonick E, Iacobucci A. Use of chemotherapy during human pregnancy. Lancet Oncol 2004;5:283-291.

39. Serrano JM, González I, Del Castillo S, et al. Diastolic dysfunction following anthracycline-based chemotherapy in breast cancer patients: incidence and predictors. Oncologist. 2015;20:864-872.

40. Cardonick E, Dougherty R, Grana G, Gilmandyar D, Ghaffar S, Usmani A. Breast cancer during pregnancy: maternal and fetal outcomes. Cancer J 2010;16:76-82. 
41. Van Calsteren K, Verbesselt R, Ottevanger N, et al. Pharmacokinetics of chemotherapeutic agents in pregnancy: a preclinical and clinical study. Acta Obstet Gynecol Scand 2010;89:1338-1345.

42. Cardonick E, Bhat A, Gilmandyar D, Somer R. Maternal and fetal outcomes of taxane chemotherapy in breast and ovarian cancer during pregnancy: case series and review of the literature. Ann Oncol 2012;23:3016-3023.

43. Zagouri F, Sergentanis TN, Chrysikos D, et al. Taxanes for breast cancer during pregnancy: a systematic review. Clin Breast Cancer 2013;13:16-23.

44. Ring AE, Smith IE, Ellis PA. Breast cancer and pregnancy. Ann Oncol 2005; 16:1855-1860.

45. Pianca N, Shafiei M, George M. Trastuzumab Exposure in Early Pregnancy for a Young Lady with Locally Invasive Breast Cancer. World J Oncol 2015;6:381-382.

46. Yildirim N, Bahceci A. Use of pertuzumab and trastuzumab during pregnancy. Anticancer Drugs 2018;29:810-813.

47. Johansson AL, Andersson TM, Hsieh CC, Cnattingius S, Lambe M. Increased mortality in women with breast cancer detected during pregnancy and different periods postpartum. Cancer Epidemiol Biomarkers Prev 2011;20:1865-7182.

48. Eniu DT, Romanciuc F, Moraru C, et al. The decrease of some serum free amino acids can predict breast can- cer diagnosis and progression. Scand J Clin Lab Invest 2019;79:17-24.

49. Genin AS, De Rycke Y, Stevens D, et al. Association with pregnancy increases the risk of local recurrence but does not impact overall survival in breast cancer: A case-control study of 87 cases. Breast 2016;30:222-227.

50. Rodriguez AO, Chew H, Cress R, et al. Evidence of poorer survival in pregnancy-associated breast cancer. Obstet Gynecol 2008;112:71-78.

51. Azim HA Jr, Santoro L, Russell-Edu W, Pentheroudakis G, Pavlidis N, Peccatori FA. Prognosis of pregnancy-associated breast cancer: a meta-analysis of 30 studies. Cancer Treat Rev 2012;38:834-842.

52. Callihan EB, Gao D, Jindal S, et al. Postpartum diagnosis demonstrates a high risk for metastasis and merits an expanded definition of pregnancy-associated breast cancer. Breast Cancer Res Treat 2013;138:549-559.

53. Froehlich K, Stensheim H, Markert UR, Turowski G. Breast carcinoma in pregnancy with spheroid-like placental metastases-a case report. APMIS 2018;126:448452.

54. Grabarz A, Azais H, Petit S, Dedet B. Placental metastasis of breast adenocarcinoma. Gynecol Obstet Fertil Senol 2017;45:323-324. 